\title{
Lesson of the month 1: Neurosyphilis mimicking viral encephalitis and ischaemic stroke
}

\author{
Authors: Jessica Green, ${ }^{A}$ Nicholas Savage, ${ }^{B}$ Colin Jenkins ${ }^{C}$ and Catherine Chima-Okereke ${ }^{D}$
}

With the incidence of syphilis more than doubling over the last decade, knowledge of its manifestations is of increasing importance. Yet, today's clinicians are less experienced in the recognition of syphilis than the physicians of Osler's day.

In this case, a 56-year-old man presented with acute confusion and a history suggestive of encephalitis.

Neuroimaging revealed cystic infarcts. He was subsequently tested for HIV and syphilis and found to be positive for both. HIV co-infection had accelerated the rate of neurosyphilis progression. After timely diagnosis and treatment of both conditions, cognitive testing returned to baseline.

This case highlights that neuroimaging can often show nonspecific infarcts and haemorrhages instead of characteristic syphilitic gummae. This variability adds to the challenge of diagnosis. Thus syphilis serology screening should be sent in those presenting with acute confusion and neuroimaging abnormalities. In cases of cryptogenic stroke, syphilis serology should be added to the screening tests.

KEYWORDS: Syphilis, encephalitis, neurosyphilis, HIV co-infection, stroke

\section{Presentation}

A retired 56-year-old man presented to the emergency department having been found wandering in the supermarket. He was confused and displaying unusual behaviour. Apart from a generalised headache over the previous 6 weeks, he had been relatively well with no significant past medical history. He usually drank a half bottle of wine and smoked three cigars daily, but denied recreational drug use.

His Glasgow coma score was 14 on account of confused speech seemingly resulting from visual hallucinations. He was otherwise neurologically intact.

Initial bloods, urinalysis and chest $\mathrm{x}$-ray were within normal limits. Computed tomography of the head showed mature bilateral basal

Authors: ${ }^{A}$ registrar stroke medicine, Wye Valley NHS Trust, Hereford, UK; ${ }^{\text {B }}$ clinical education fellow, Wye Valley NHS Trust, Hereford, UK; ' Consultant stroke physician, Wye Valley NHS Trust, Hereford, UK; ${ }^{\text {D }}$ consultant in sexual health, Worcestershire Health and Care NHS Trust, Hereford, UK

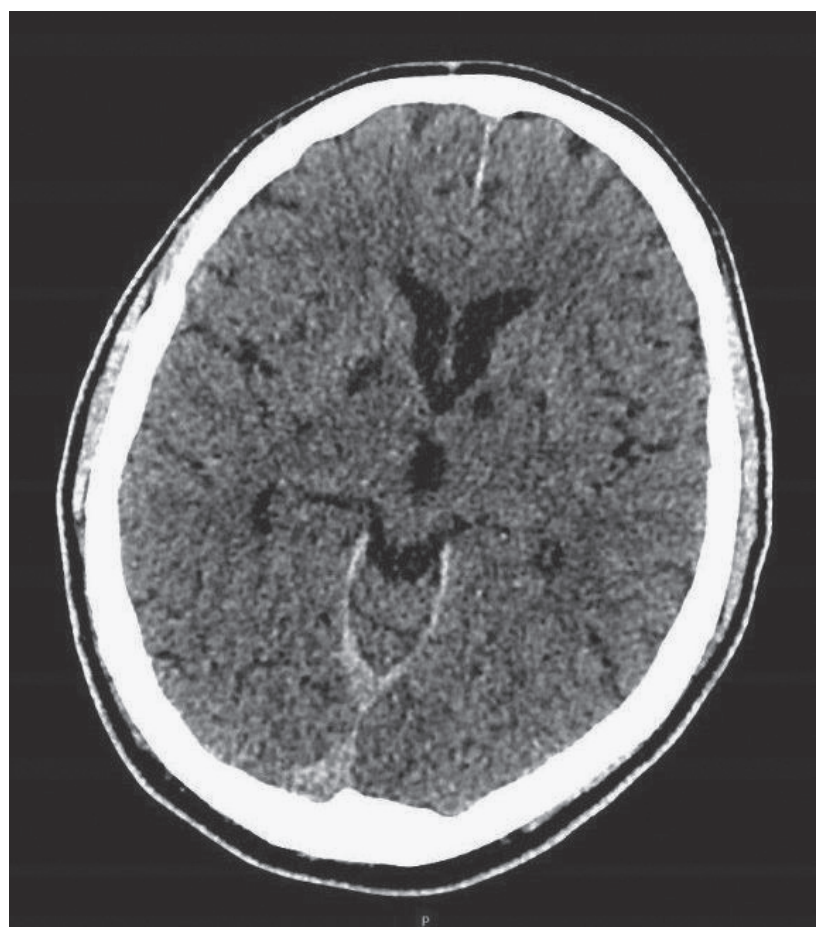

Fig 1. Computed tomography of the head.

ganglia infarcts (see Fig 1). Due to a presentation suggestive of viral encephalitis, he was started on intravenous (IV) aciclovir. High dose aspirin was also commenced.

Initially it was thought that the infarcts were merely incidental findings. Magnetic resonance imaging of the head was then requested to further characterise the unusual appearance of these lesions. This showed multiple areas of restricted diffusion (see Fig 2). These findings confirmed acute infarcts with no evidence of haemorrhage. There was a cystic appearance to the subcortical infarcts on fluid-attenuated inversion recovery (see Fig 3).

Further magnetic resonance angiography of the head showed no occlusion or dissection from aortic arch to intracranial vessels.

Despite the location of the infarcts, cognition was solely affected. On the Montreal Objective Cognitive Assessment (MOCA) tool, he scored 19/30 with recall and orientation most affected. Cerebrospinal fluid (CSF) analysis showed a leucocytosis of $7.3 \times 10^{6} / \mathrm{L}$ with elevated protein of $2,558 \mathrm{mg} / \mathrm{dL}$. 


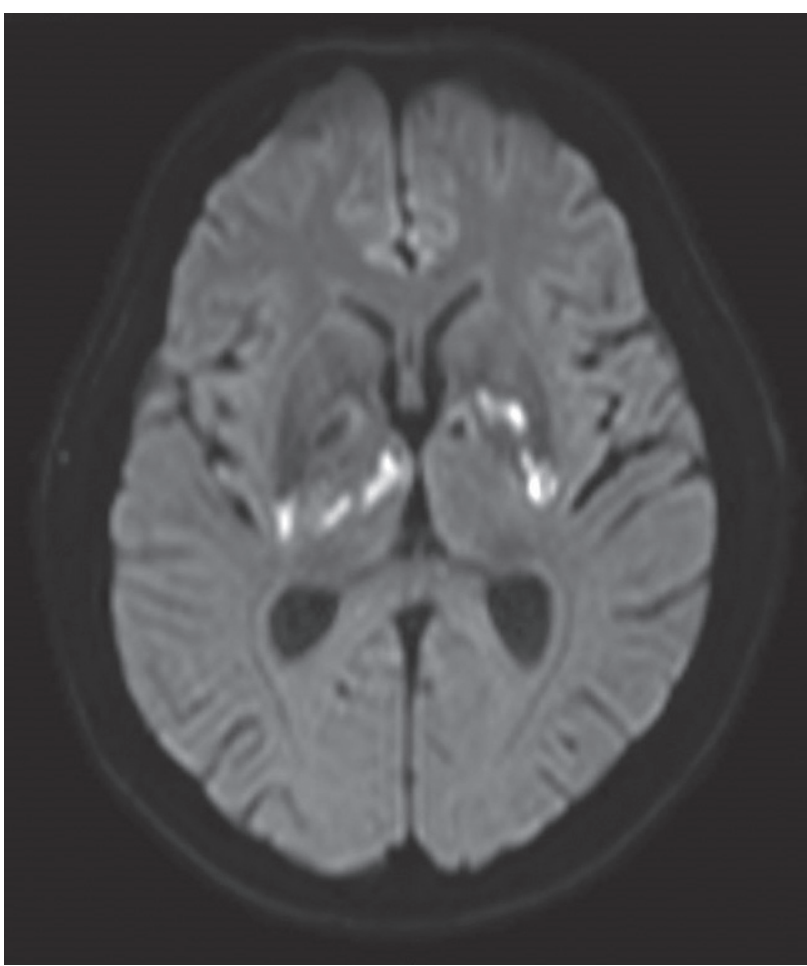

Fig 2. Diffusion-weighted magnetic resonance imaging of the head.

Polymerase chain reaction for herpes simplex virus was negative and there was no clinical improvement on IV aciclovir after 72 hours.

Further history taking revealed a homosexual encounter in Amsterdam. A wider net of investigations was cast to include HIV and syphilis serology. HIV 1 and 2 were confirmed with an HIV viral load of 413,000 copies/mL. Lymphopenia of $1.5 \times 10^{9} / \mathrm{L}$ was noted during admission. CD4 cell count showed a proportionally low differential of $15 \%\left(204\right.$ cells $/ \mathrm{mm}^{3}$ ) vs CD8 $69 \%\left(935\right.$ cells $\left./ \mathrm{mm}^{3}\right)$.

Upon further questioning, the patient reported a transient period of malaise and mild fever 16 weeks previously. This likely represents seroconversion. All three syphilis serological markers were positive; rapid plasma reagin (RPR) at $>1: 64$, treponema pallidum particle agglutination assay (TPPA) at >1:5,120 and syphilis immunoglobulin (Ig) M/IgG. CSF RPR and CSF TPPA were reactive at $>1: 32$ and 1:2,560, respectively, thus confirming neurosyphilis.

IV benzylpenicillin was commenced for 14 days. On the third day, he began to show significant improvement and on the eleventh day his MOCA improved to $26 / 30$ with orientation now back at baseline, but ongoing effects on long-term recall.

At 6-month follow-up, he remained on clopidogrel $75 \mathrm{mg}$ daily, dolutegravir $50 \mathrm{mg}$ daily (an integrase inhibitor) and truvada 200/245 mg daily (double nucleoside reverse transcriptase inhibitors). His HIV was well controlled with the latest CD4 cell count 395 cells $/ \mathrm{mm}^{3}$ (18\%) and HIV viral load 195 copies/ $\mathrm{mL}$. RPR titre had dropped from >1:64 to 1:16 with treatment, which is indicative of a good response to syphilis treatment. MOCA returned to baseline showing no lasting sequelae of neurosyphilis.

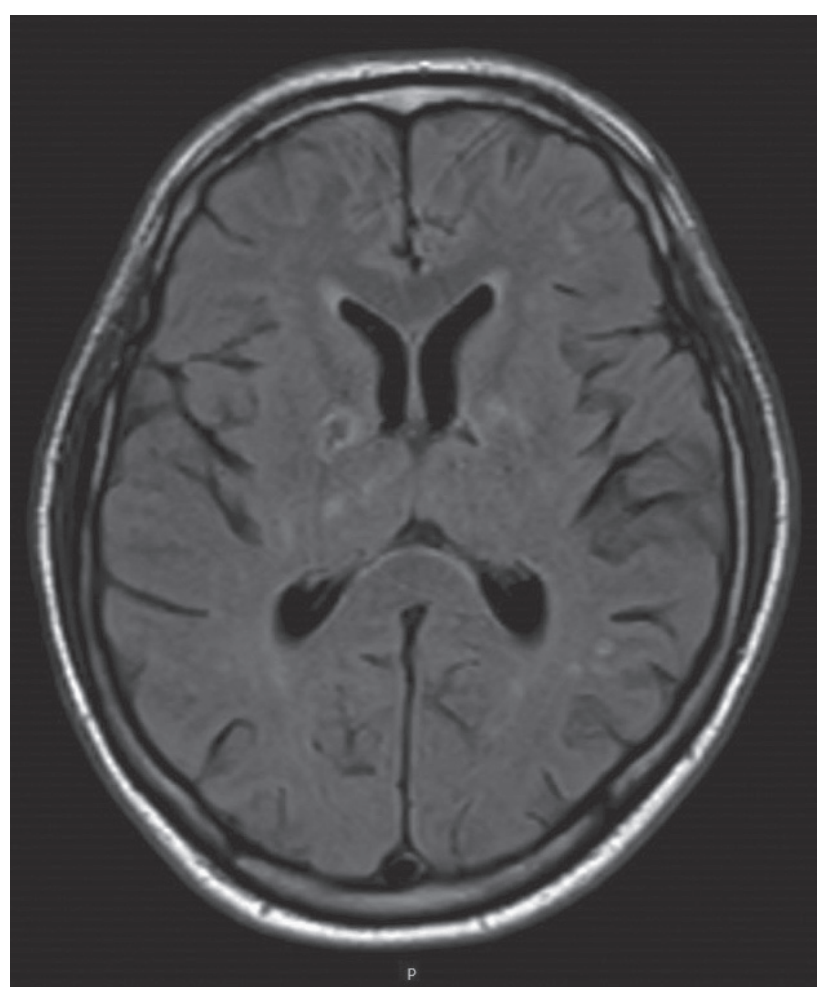

Fig 3. Fluid-attenuated inversion recovery magnetic resonance imaging of the head.

\section{Discussion}

With a $144 \%$ increase in incidence of syphilis from 2008 to 2017 (5.5 per 100,000 population increasing to 12.7 per 100,000 population), ${ }^{1}$ the diagnosis of syphilis in its many manifestations needs once again to be at the forefront of the clinician's mind.

With concurrent HIV infection, syphilis is likely to progress to the meningovascular form of neurosyphilis at a faster rate ${ }^{2}$ (approximately only 16 weeks in our case). This would mean faster progression to the sequelae of syphilis infection and thus the need for diagnosis becomes even more urgent. If syphilis or HIV is confirmed on testing, it is prudent to test for the other condition as they have an increased chance of concurrent infection. ${ }^{3}$

Unfortunately the neuroimaging findings of syphilis are often non-specific, such as infarct and haemorrhage rather than the classic syphilitic gummae. ${ }^{4}$ This leaves a high likelihood of such patients being mistaken for someone who has developed cognitive impairment post-stroke. Such a wide variation in neuroimaging findings makes the diagnosis of neurosyphilis challenging.

In a study of 149 patients where ischaemic stroke is the presenting symptom, there is a high rate of syphilis misdiagnosis $(80.95 \%) .{ }^{5}$ Liu et al noted that all neurosyphilis confirmed following a stroke had only ever been identified at follow-up. This may be because there is such a wide differential to consider in presentations of cryptogenic stroke.

To clear treponema pallidum from the CSF IV benzylpenicillin $1.8-2.4 \mathrm{~g}$ should be given every 4 hours for a fortnight while an inpatient. Once confusion has settled, the other option is to treat 
as an outpatient with daily intramuscular injection of procaine penicillin and oral probenicid four times a day. ${ }^{6}$

When a patient presents with confusion, and encephalitis is suspected, HIV and syphilis serology should be added to the screen. This may be particularly useful when sexual history is not forthcoming or even impossible to ascertain due to acute confusion.

\section{References}

1 Public Health England. Table 1: STI diagnoses \& rates in England by gender, 2008-2017. London: PHE, 2018. https://assets.publishing. service.gov.uk/government/uploads/system/uploads/attachment_data/ file/713276/2017_Table_1_STI_diagnoses_rates_in_England_by_ gender.pdf [Accessed 12th October 2018].

2 Patira R, Smith-Benjamin S, Wang J. Stroke in a young patient with neurosyphilis and HIV. Int J STD AIDS 2017;28:306-9.
3 Firlag-Burkacka E, Swiecki P, Cielniak I et al. High frequency of neurosyphilis in HIV-positive patients diagnosed with early syphilis. HIV Med 2016:17:323-6.

4 Czarnowska-Cubała M, Wiglusz M, Cubała W et al. MR findings in neurosyphilis - A literature review with a focus on a practical approach to neuroimaging. Psychiatria Danubina 2013;25(Suppl 2):S153-7.

5 Liu LL, Zheng WH, Tong ML et al. Ischaemic stroke as a primary symptom of neurosyphilis among HIV-negative emergency patients. J Neurol Sci 2012;317:35-9.

6 Kingston M, French P, Higgins S. UK National Guidelines on the management of syphilis 2015. Int J STD AIDS 2015;0:1-26.

Address for correspondence: Dr Jessica Green, Postgraduate Centre, Hereford County Hospital, Hereford HR1 2BN, UK. Email: jcpgreen@doctors.org.uk

\section{Looking for jobs in medicine?}

\section{Medicine Jobs is the RCP's recruitment website, providing doctors with a new way to search for their next career move.}

\section{Find your next hospital job}

Visit Medicine Jobs to find the latest NHS vacancies for consultant, specialty and associate specialist (SAS), physician associate and trust grade posts.

Find out more and subscribe to the monthly Medicine Jobs bulletin at www.rcplondon.ac.uk/jobsboard 